\title{
POINTWISE DEFINABLE MODELS OF SET THEORY
}

\author{
JOEL DAVID HAMKINS, DAVID LINETSKY, AND JONAS REITZ
}

\begin{abstract}
A pointwise definable model is one in which every object is definable without parameters. In a model of set theory, this property strengthens $V=\mathrm{HOD}$, but is not first-order expressible. Nevertheless, if ZFC is consistent, then there are continuum many pointwise definable models of ZFC. If there is a transitive model of ZFC, then there are continuum many pointwise definable transitive models of ZFC. What is more, every countable model of ZFC has a class forcing extension that is pointwise definable. Indeed, for the main contribution of this article, every countable model of GödelBernays set theory has a pointwise definable extension, in which every set and class is first-order definable without parameters.
\end{abstract}

\section{INTRODUCTION BY WAY OF A CURIOUS LOGIC CONUNDRUM}

One occasionally hears the argument-let us call it the math-tea argument, for perhaps it is heard at a good math tea - that there must be real numbers that we cannot describe or define, because there are are only countably many definitions, but uncountably many reals. Does it withstand scrutiny? ${ }^{1}$

We can be precise, of course, and define that an element $a$ of a structure $\mathcal{M}$ is definable (without parameters) when there is a formula $\varphi(x)$ in the language of $\mathcal{M}$ such that $\mathcal{M} \models \varphi[x]$ only at $x=a$. In the continuum of the ordered real line $\langle\mathbb{R},<\rangle$, for example, there are no definable elements, since the automorphism group acts transitively by translation and all points look alike. With the ordered field structure $\langle\mathbb{R},+, \cdot, 0,1,<\rangle$, however, every algebraic number becomes definable,

2000 Mathematics Subject Classification. 03E55.

Key words and phrases. set theory, forcing.

The research of the first author has been supported in part by grants from the National Science Foundation, the CUNY Research Foundation and the Simons Foundation. The research of the third author has been supported in part by grants from the CUNY Research Foundation.

${ }^{1}$ See [Ani] for an instance of the argument at MathOverflow, which surely serves a brisk cup of math tea online. We leave aside the remark of Horatio, eight-year-old son of the first author, who announced, "Sure, papa, I can describe any number. Let me show you: tell me any number, and I'll tell you a description of it!" 
but only these, by Tarski's theorem on real closed fields. As one adds additional structure $\left\langle\mathbb{R},+, \cdot, 0,1,<, \mathbb{Z}, \sin (x), e^{x}, \cdots\right\rangle$, the collection of definable reals grows larger. Eventually, we will in our definitions be attracted to the possibilities of using higher order mathematical objects and constructions, such as function classes, spaces or measures, and this amounts to defining objects in increasingly large fragments $V_{\alpha}$ of the set-theoretic universe. Most all of the classical mathematical structure is itself definable in the set-theoretic structure $\left\langle V_{\omega+\omega}, \in\right\rangle$, a model of the Zermelo axioms, and so the definable reals of this structure includes almost every real ever defined classically. The structures arising with larger ordinals, however, allow us to define even more reals.

It would be a kind of cheating, for the problem at hand, to define a real $r$ or other object by using a language or structure that was itself otherwise undefinable or uncanonical, such as by using a constant symbol with value $r$ or a unary predicate satisfied only at $r$; this would be like defining $\pi$ as "the value of the constant symbol $\pi$," which is surely unsatisfying. Similarly, we do not want to offer a definition of $r$ in some enormous $\left\langle V_{\alpha}, \in\right\rangle$ when $\alpha$ is not itself somehow definable, since in effect this is using $\alpha$ as a kind of parameter. Rather, the spirit of the problem seems to be to define reals using only structure that is itself definable in the set-theoretic background. This amounts, of course, simply to defining the real directly with respect to the set-theoretic background $\langle V, \in\rangle$ in the first place.

In any fixed structure $\mathcal{M}$ in a countable language, including the higher-order set-theoretic structures $\left\langle V_{\alpha}, \in\right\rangle$, the math-tea argument seems fine: since there are only countably many definitions to use, but uncountably many reals, there will indeed be many reals that are not definable there.

But when we make the move as we have discussed to defining reals or other objects with respect to the set-theoretic background $\langle V, \in\rangle$, a subtle meta-mathematical obstacle arises for the math-tea argument. Specifically, in order to count the definable objects, the argument presumes that we have a way of associating to each definable object a definition of it, a definability map $r \mapsto \psi$, where $r$ is the unique object satisfying $\psi(r)$. This is not a problem when we are working with definitions over a set structure, since the satisfaction relation over a set structure is definable from that structure, but it becomes problematic with definitions over $V$, for reasons connected with Tarski's theorem on the non-definability of truth. Basically, there is no uniform first-order way to express the concept " $x$ is defined by formula $\psi$ " within set theory. This obstacle for the math-tea argument suggests a possibility 
that perhaps one could live in a model of ZFC in which every real is definable without parameters.

Question 1. Is it consistent with the axioms of set theory that every real is definable in the language of set theory without parameters?

The answer is Yes. Indeed, much more is true: if the ZFC axioms of set theory are consistent, then there are models of ZFC in which every object, including every real number, every function on the reals, every set of reals, every topological space, every ordinal and so on, is uniquely definable without parameters. Inside such a universe, the math-tea argument comes ultimately to a false conclusion.

Definition 2. A first-order structure $\mathcal{M}$ is pointwise definable if every element of $\mathcal{M}$ is definable in $\mathcal{M}$ without parameters.

In a pointwise definable model, every object can be specified as the unique object with some first-order property. In such models, all objects are discernible; every object satisfies a unique principal complete 1-type. Notice that the property of being pointwise definable is not first-order expressible, since it is not preserved by elementary extensions. Clearly, pointwise definable models in a countable language must be countable, since there are only countably many definitions.

\section{Theorem 3.}

(1) If $\mathrm{ZFC}$ is consistent, then there are continuum many nonisomorphic pointwise definable models of $\mathrm{ZFC}$.

(2) If there is a transitive model of ZFC, then there are continuum many transitive pointwise-definable models of ZFC.

(3) Every countable model of ZFC has a class forcing extension that is pointwise definable.

(4) Every countable model of GBC has a pointwise definable extension, in which every set and class is first-order definable without parameters.

The first two claims have fairly soft proofs and might be considered to be a part of the mathematical folklore. Statement (1) could be credited to Myhill [Myh52]. The latter two claims are more substantial forcing arguments. Statement (3) is mentioned independently in [Ena05] and, in the case of countable transitive models, in [Dav82]. Our main contribution is statement (4), which implies the earlier statements. The rest of this article is devoted to proving these facts and several other related results we find interesting. We begin in section 2 with some elementary observations about pointwise definable models of set theory and prove the easier initial results. In sections 3 and 4, 
we move into the forcing arguments, relying on work of Simpson in the case of ZFC and a result of S. Friedman, building on a result of Kossak and Schmerl from PA, for the fully general GBC case.

\section{Pointwise Definable Models of ZFC}

The first task is to establish the basic fact that pointwise definable models of set theory do indeed exist.

Theorem 4. If there is a model of $\mathrm{ZFC}$, then there is a pointwise definable model of ZFC. Indeed, there are continuum many non-isomorphic such models.

Proof. If there is a model of ZFC, then there is a model $M \models \mathrm{ZFC}+$ $V=$ HOD. Such a model has a parameter-free definable well-ordering of the universe, and therefore it has parameter-free definable Skolem functions, which simply select the least witness with respect to the definable well-ordering. If $M_{0}$ is the collection of all definable elements of $M$, then it is closed under these definable Skolem functions and thus $M_{0} \prec M$. From this, it follows that definitions work the same in $M_{0}$ as in $M$, and so every object of $M_{0}$ is definable in $M_{0}$. Thus, $M_{0}$ is a pointwise definable model of ZFC. By the Gödel-Rosser theorem, there are continuum many consistent completions of ZFC $+V=\mathrm{HOD}$, and we have established that each such theory has a pointwise definable model.

Myhill [Myh52] was evidently the first to observe (in contemporary language) that if $\mathrm{ZFC}$ is consistent, then there is a pointwise definable model of $\mathrm{GBC}+V=L$, by essentially the argument we have given, using the definable Skolem functions of $L$. Indeed, Myhill was fully aware of the implications for the math-tea argument, for he concludes his otherwise terse article with:

One often hears it said that since there are indenumerably many sets and only denumerably many names, therefore there must be nameless sets. The above shows this argument to be fallacious.

The fact is that every pointwise definable model of ZFC arises in exactly the manner of the proof of theorem 4. A prime model is one that embeds elementarily into every model of its theory.

Observation 5. The following are equivalent:

(1) $M$ is a pointwise definable model of ZFC.

(2) $M$ consists of the definable elements, without parameters, of a model of $\mathrm{ZFC}+V=\mathrm{HOD}$. 
(3) $M$ is a prime model of $\mathrm{ZFC}+V=\mathrm{HOD}$.

Proof. The first statement implies the second since every pointwisedefinable model satisfies $V=\mathrm{HOD}$, and the converse implication is the proof of theorem 4 . These models are exactly the prime models of $\mathrm{ZFC}+V=\mathrm{HOD}$, since the theory admits definable Skolem functions, and so every model of $\mathrm{ZFC}+V=\mathrm{HOD}$ elementarily embeds the definable hull of the empty set in that model.

More generally, if $M$ is any model of ZF, then the collection $M_{0}$ consisting of the elements of $M$ that are definable without parameters in $M$ is an elementary substructure of $\mathrm{HOD}^{M}$, because it is closed under the canonical Skolem functions for $\mathrm{HOD}^{M}$, which are definable in $M$. A similar fact is observed in [Ena05, Theorem 2.11]. The model $M_{0}$ is therefore prime relative to $M$, in the sense that whenever $M \equiv N$, then $M_{0}$ embeds elementarily in $\mathrm{HOD}^{N}$.

Note that if two pointwise definable models have the same theory, then they are isomorphic, since the definitions of the elements tell you the isomorphism. Next, we show that there are also numerous wellfounded pointwise definable models of set theory, if there is a wellfounded model of set theory at all.

Theorem 6. If there is a transitive model of ZFC, then there are continuum many transitive pointwise-definable models of ZFC.

Proof. If there is a transitive model $M$ of $\mathrm{ZFC}$, then there is a transitive model $N$ of ZFC $+V=$ HOD, such as the model $L^{M}$ or any of the models obtained by forcing $V=$ HOD over $M$, and such models exist if $M$ is countable. By observation 5, the collection of parameter-free definable elements of $N$ is a well-founded pointwise-definable elementary substructure, whose Mostowski collapse is as desired.

To construct continuum many such models, suppose that $M$ is any countable transitive model of ZFC. Since $M$ is countable, there are a perfect set of $M$-generic Cohen reals $c$, with which we may form the forcing extension $M[c]$. By any of the usual methods, such as coding sets into the GCH pattern (which we will shall explain in greater detail in section 3), we may form a further forcing extension $M[c][G]$ satisfying $\mathrm{ZFC}+V=\mathrm{HOD}$. The $M[c]$-generic filter $G$ is built in a diagonal construction meeting the countably many dense classes of $M[c]$. By ensuring that $c$ is the first set to be coded into the GCH pattern -we could arrange that the GCH holds in $M[G]$ at $\aleph_{n}$ exactly when the $n^{\text {th }}$ digit of $c$ is 1 -we may assume that $c$ is definable without parameters in $M[c][G]$. Thus, if $M_{0}$ is the collection of definable elements of $M[c][G]$, then it is pointwise definable and contains $c$. Since there are 
continuum many such reals $c$, we have thereby produced continuum many transitive pointwise definable models of ZFC.

A similar observation establishes the following folklore result. The minimal transitive model of $\mathrm{ZFC}$ is $L_{\alpha}$ for the smallest ordinal $\alpha$ for which $L_{\alpha} \models \mathrm{ZFC}$, if any such ordinal exists. If $M$ is any transitive model of ZFC, then $L^{M} \models \mathrm{ZFC}$ and $L^{M}=L_{\alpha}$ for $\alpha=\mathrm{ORD}^{M}$, and so the minimal model exists and is included within $M$, thereby justifying its name.

Theorem 7. The minimal transitive model of ZFC is pointwise definable. If $L_{\beta}$ is pointwise definable, then the next $\hat{\beta}>\beta$ with $L_{\hat{\beta}}=\mathrm{ZFC}$, if it exists, is also pointwise definable.

Proof. If $L_{\alpha}$ is the minimal transitive model of ZFC, then by condensation the definable hull of $\emptyset$ in $L_{\alpha}$ collapses to $L_{\alpha}$, and so every element of $L_{\alpha}$ is definable in $L_{\alpha}$. If $L_{\beta}$ is a pointwise definable model of ZFC and $\hat{\beta}$ is the smallest ordinal above $\beta$ for which $L_{\hat{\beta}} \models \mathrm{ZFC}$, then $\beta$ is definable in $L_{\hat{\beta}}$ as the largest ordinal such that $L_{\beta} \models$ ZFC. It follows that every element of $L_{\beta}$ is definable in $L_{\hat{\beta}}$, by using a definition relativized to $L_{\beta}$, which is definable in $L_{\hat{\beta}}$. If $D$ is the set of definable elements of $L_{\hat{\beta}}$, then the Mostowski collapse of $D$ will have height larger than $\beta$, and be a model of ZFC $+V=L$, so by the minimality of $\hat{\beta}$ it will be all of $L_{\hat{\beta}}$. Thus, $L_{\hat{\beta}}$ is pointwise definable, as desired.

The phenomenon of theorem 7 extends to describable limits of such $L_{\alpha}$. For example, the first $L_{\beta} \models \mathrm{ZFC}$ for which $\beta$ is a limit of $\alpha$ for which $L_{\alpha} \models$ ZFC is also pointwise definable, because the definable hull of $L_{\beta}$ will collapse to such a limit and therefore collapse to $L_{\beta}$ itself. And similarly for many other limits. But if $L_{\alpha}$ is an elementary substructure of $L_{\beta}$, for $\alpha \neq \beta$, then of course $L_{\beta}$ cannot be pointwise definable, since the definable elements must lie in the range of the embedding. Similarly, if $\omega_{1}^{L} \leq \alpha$, then $L_{\alpha}$ cannot be pointwise definable, irrespective of whether it satisfies ZFC or not, since this property is absolute to $L$ and such $L_{\alpha}$ are uncountable in $L$.

Theorem 8. There are arbitrarily large $\xi<\omega_{1}^{L}$ for which $\left\langle L_{\xi}, \epsilon\right\rangle$ is pointwise definable. If there are arbitrarily large $\alpha<\omega_{1}^{L}$ for which $L_{\alpha} \models \mathrm{ZFC}$, then there are arbitrarily large such $\alpha$ for which $L_{\alpha} \models \mathrm{ZFC}$ and is pointwise definable.

Proof. The second claim is essentially [Ena02, thm 3.7]. For the first claim, observe that every real of $L$ is definable without parameters in some countable $L_{\xi}$, because the $L$-least real $z$ not definable in any 
countable $L_{\xi}$, if any should exist, is thereby definable in $L_{\omega_{1}^{L}}$, which will condense to a definition of $z$ in some countable $L_{\xi}$, giving a contradiction. Indeed, by taking the definable hull of the empty set, we find that $z$ is definable in a pointwise definable $L_{\xi}$. It follows that such $\xi$ must be unbounded in $\omega_{1}^{L}$, establishing the first claim. For the second claim, suppose that there are unboundedly many $\alpha<\omega_{1}^{L}$ for which $L_{\alpha}=$ ZFC. Observe that every countable ordinal $\xi<\omega_{1}^{L}$ is definable without parameters in $L_{\alpha} \models$ ZFC, where $\alpha$ is the $(\xi+1)^{\text {th }}$ ordinal for which $L_{\alpha} \models$ ZFC, because this $L_{\alpha}$ can see exactly $\xi$ many smaller $\beta$ for which $L_{\beta}=\mathrm{ZFC}$, an argument that recalls the definition of Laver functions for uplifting cardinals in [HJ]. Combining the previous facts, every real $z$ of $L$ is definable in some $L_{\xi}$, which is itself definable in some $L_{\alpha} \models$ ZFC, and so $z$ is in the definable hull of $L_{\alpha}$, which condenses to a pointwise definable $L_{\alpha_{0}} \models$ ZFC containing $z$. Since $z$ was arbitrary in $L$, these $\alpha_{0}$ must be unbounded in $\omega_{1}^{L}$, as desired.

A model $M$ of $\mathrm{ZF}$ is $\omega$-standard if the natural numbers of $M$ have the standard order-type $\omega$. More generally, $M$ is $\zeta$-standard for an ordinal $\zeta$ if the ordinals of $M$ have a $\zeta^{\text {th }}$ member, so that the well-founded initial segment of the ordinals of $M$ has order type exceeding $\zeta$.

Theorem 9. If there are arbitrarily large $\alpha<\omega_{1}^{L}$ for which $L_{\alpha} \models \mathrm{ZFC}$, then every real in $V$ is an element of a pointwise definable $\omega$-standard model of $\mathrm{ZFC}+V=L$, whose well-founded initial segment can be as large in the countable ordinals of $V$ as desired.

Proof. The argument of theorem 8 shows under the hypothesis that the conclusion is true in $L$. We complete the proof of the theorem by observing that the statement that every real of $V$ is in a pointwise definable $\omega$-standard model of $\mathrm{ZFC}+V=L$ has complexity $\Pi_{2}^{1}$, since it has the form "for every real, there is a countable structure...," where the omitted portion is arithmetic, since it involves quantification only over the elements of the structure and the digits of the real. Thus, by Shoenfield absoluteness this statement is absolute from $L$ to $V$ and hence is true in $V$, as desired, even if $V$ has many more reals and larger countable ordinals than $L$. Note that if an $\omega$-standard model $M$ has a real $z$ coding a countable ordinal $\zeta$, then $M$ will decode this real correctly, and so $M$ will be well-founded beyond $\zeta$, and the theorem is proved.

Corollary 10. If there are arbitrarily large $\alpha<\omega_{1}^{L}$ with $L_{\alpha} \models \mathrm{ZFC}$, then every countable transitive set $M$ is a countable transitive set inside a structure $M^{+}$that is a pointwise definable model of $\mathrm{ZFC}+V=L$, and $M^{+}$is well-founded beyond the rank of $M$. 
Proof. Code $M$ by a real $z$, and place $z$ inside a pointwise definable $\omega$-standard model $M^{+} \models \mathrm{ZFC}+V=L$. Since $M^{+}$performs the Mostowski collapse of the structure coded by $z$ correctly, it follows that $M$ is a transitive set in $M^{+}$. (In this sense, $M$ end-extends to $M^{+}$.) In particular, $M^{+}$is well-founded beyond the rank of $M$.

Let us notice a few things about these arguments. First, although in $L$ we produced fully well-founded pointwise definable models, if in theorem 9 we were to have made that part of the statement, then the complexity would have risen to $\Pi_{3}^{1}$ and we would have lost the absoluteness that allowed us to bring the statement from $L$ to $V$. And clearly, we cannot expect to place non-constructible reals inside wellfounded models of $V=L$. Second, the hypothesis that there are arbitrarily large $\alpha<\omega_{1}^{L}$ with $L_{\alpha} \models$ ZFC follows from the simpler (but strictly stronger) hypothesis that there is a single uncountable transitive model of ZFC, because one may consider increasingly large countable elementary substructures of the $L$ of such a model. We may omit these hypotheses completely in the theorems above, if it is desired only to have $\omega$-standard pointwise definable models of $\mathrm{ZFC}^{*}+V=L$, where $\mathrm{ZFC}^{*}$ is some finite fragment of $\mathrm{ZFC}$, rather than full ZFC, since by reflection and condensation there are arbitrarily large countable $L_{\alpha}$ satisfying any such finite fragment $\mathrm{ZFC}^{*}$.

The results become rather curious when there are reals in $V$ that are not in $L$. Suppose there are sufficient $L_{\alpha} \models$ ZFC and we force to collapse $\omega_{1}$, with the resulting forcing extension $V[g]$, where $g$ is a real coding $\omega_{1}^{V}$. By the argument above, there is in $V[g]$ a countable $\omega$-standard model $M \models \mathrm{ZFC}+V=L$ in which $g$ exists and is actually definable. Since $M$ has the standard $\omega$, it interprets $g$ correctly and so the well-founded part of $M$ exceeds $\omega_{1}^{V}$. But since $M \models V=L$, it thinks that $g$ is constructible as well as definable, constructed at some (nonstandard) ordinal stage $\tilde{\alpha}>\omega_{1}^{V} \geq \omega_{1}^{L}$ !

Another interesting example arises when $0^{\sharp}$ exists. This assumption implies the hypothesis of the theorem, and so by the theorem there is a pointwise definable model $M$ of $\mathrm{ZFC}+V=L$, well-founded as high in the countable ordinals as desired, in which the true $0^{\sharp}$ is a member, thought by $M$ to be constructible at some stage $\tilde{\alpha}$, necessarily in the illfounded part of $M$. Thus, the true $0^{\sharp}$ exists unrecognized but definable in a model of $\mathrm{ZFC}+V=L$ that is well-founded far beyond the true $\omega_{1}^{L}$ ! For example, the model $M$ can be well-founded beyond a rich class of (countable) Silver indiscernibles (or even beyond $\omega_{1}^{V}$ if one forces to collapse as in the previous paragraph) and have the same theory as the 
true $L^{V}$. The true ordinal indiscernibles of $V$ become discernible in $M$, however, since it is pointwise definable.

Corollary 10 may seem paradoxical when applied to a countable transitive model $M$ having enormous large cardinals incompatible with $L$. For example, perhaps $M$ has supercompact cardinals inside it or has measures that are iterable in $V$, meaning all iterates are well-founded; they cannot be iterable in $M^{+}$, since this is a model of $V=L$. In any case, corollary 10 already implies that every countable model of ZFC or GBC admits an end-extension to a pointwise definable model of set theory. In theorems 11 and 13, however, we shall find pointwise definable extensions with the same ordinals.

Let us now turn to the question of the extent to which definability is first-order expressible, by making a number of observations that illustrate the range of possibility. We have already noted that the property of a model being pointwise definable is not first-order expressible, since it is not preserved by nontrivial elementary extensions. Since pointwise definability is a strong generalization of the axiom $V=$ HOD, it is tempting to introduce such notation as $V=D$ or $V=H D$ to express that a model is pointwise definable, thereby maintaining a parallel to the classical $V=$ HOD notation while emphasizing that the definitions need no parameters. We hesitate to adopt this notation, however, because we fear it would incorrectly suggest that the concept is first-order expressible, which isn't the case.

(i) There is no uniform definition of the class of definable elements. Specifically, there is no formula $\mathrm{df}(x)$ in the language of set theory that is satisfied in any model $M \models$ ZFC exactly by the definable elements. The reason is that if $M_{0}$ is pointwise definable and $M_{0} \prec M$ is a nontrivial elementary extension, then the definable elements of $M_{0}$ and $M$ are precisely the elements of $M_{0}$, and so $M_{0}$ should satisfy $\forall x \operatorname{df}(x)$ but $M$ would satisfy $\exists x \neg \operatorname{df}(x)$, contrary to $M_{0} \prec M$.

(ii) In some models of set theory, the class of definable elements is a definable class. Although there is no uniform definition of the class of definable elements, it can sometimes happen that a model enjoys a certain structure that allows it to see its collection of definable elements as a definable class. For example, in a pointwise definable model, the class of definable elements includes every object and is therefore defined by the formula $x=x$. See also (iv) and (v) below.

(iii) In other models, the collection of definable elements is not a class. Consider any pointwise definable model $M \models \mathrm{ZFC}$, and let $N$ be an ultrapower of $M$ by a nonprincipal ultrafilter. The parameterfree definable elements of $N$ are exactly the elements in the range of the 
embedding. If this collection were a class in $N$ then $N$ could reconstruct $M$ and realize itself as an ultrapower, which is impossible.

(iv) In some models, the definable elements form a definable class, but there is no class function $r \mapsto \psi_{r}$ mapping definable elements to definitions of them. Suppose that $M$ is a pointwise definable model of ZFC. The definable elements of $M$ are all of $M$, which is certainly a definable class in $M$. But $M$ cannot have a function $r \mapsto \psi_{r}$ associating to each element $r$ of $M$, or even to each real of $M$, a defining formula $\psi_{r}$ of $r$, since such a map would reveal to $M$ that it has only countably many objects.

(v) In other models, the definable elements are a set and there is a set definability map $r \mapsto \psi_{r}$. Suppose that $\kappa$ is an inaccessible cardinal (this hypothesis can be reduced), and observe by a Lowenheim-Skolem argument that there are numerous $\gamma<\kappa$ with $V_{\gamma} \prec V_{\kappa} \models$ ZFC. It follows that the definable elements of $V_{\kappa}$ are all in $V_{\gamma}$ and satisfy the same definitions there as in $V_{\kappa}$. Since $V_{\gamma}$ is a set in $V_{\kappa}$, we may construct in $V_{\kappa}$ the function $r \mapsto \psi_{r}$ that maps every definable element $r$ of $V_{\gamma}$ to the smallest definition $\psi_{r}$ of it, and because $V_{\gamma} \prec V_{\kappa}$, this function has the same property with respect to $V_{\kappa}$, as desired. The large cardinal hypothesis can be reduced; it is sufficient to have an $\omega$-model $M$ with some $M_{0} \in M$ having $M_{0} \prec M$.

(vi) No model can have a definable definability map $r \mapsto \psi_{r}$. If such a map were definable, then since there are only countably many definitions $\psi_{r}$, we could easily diagonalize against it to produce a definable real not in the domain of the map. In $(\mathrm{v})$, the map is definable from parameter $\gamma$.

The surviving content of the math-tea argument seems to be the observation that in any model with access to a definability map $r \mapsto \psi_{r}$, the definable reals do not exhaust all the reals.

\section{Pointwise Definable Forcing extensions}

Let us now move beyond the elementary methods and results of section 2, and prove that every countable model $M$ of ZFC has a carefully chosen class forcing extension $M[G]$ that is pointwise definable, so that all objects of $M$, as well as those in $M[G]$, become definable in $M[G]$ without parameters. The forcing is sufficiently adaptable so as to preserve all the usual large cardinal axioms.

Theorem 11. Every countable model of ZFC has a pointwise definable class forcing extension.

After proving this theorem (on a New York City subway platform), we came later to learn of earlier work achieving it. The introduction of 
[Dav82], for example, claims the result in the special case of countable transitive models as a corollary to its main theorem, but the article unfortunately provides little further explanation. ${ }^{2}$ Also, Ali Enayat in remark 2.8.1 of [Ena05] briefly suggests a proof method very similar to our proof of theorem 11, although he does not state the result as a formal theorem. Most of Enayat's excellent paper is concerned with the Paris models, models of ZF in which every ordinal is definable without parameters, but in several instances he achieves this by establishing pointwise definability. One of his main results is [Ena05, theorem 2.19], which asserts that if there is an uncountable transitive model of ZFC, then for every infinite cardinal $\kappa$, there is a Paris model of ZF having size $\kappa$. These are very large models, but have only countably many ordinals, since each ordinal is definable without parameters.

Our proof of theorem 11 is based on a technique of Simpson [Sim74], an early application of forcing to the study of models of arithmetic. Namely, Simpson proved that every countable model $M$ of PA or ZFC has an amenable class $U$ (i.e. for any $x \in M, x \cap U \in M$ ) with the property that every element of the expanded structure $\langle M, U\rangle$ is definable without parameters. Simpson uses forcing to define a new generic proper class $U$ which codes every element of the universe. The original structure is expanded by adding a predicate for this new class, producing a pointwise definable model in the larger language. Our strategy will be simply to follow this expansion by further forcing that codes $U$ and the new generic filter into the first-order structure of the model, thereby eliminating the need for the additional predicate $U$ and producing a pointwise definable model in the original pure language of set theory. In other words, we will show that every countable model of ZFC can be extended to a pointwise definable model of ZFC, a model in which every set is definable without parameters.

We begin with a brief review of Simpson's [Sim74] result. Although his main result concerned models of PA, he concludes his paper with a ZFC version of the theorem, and the proof given below is a straightforward adaptation of his argument to the ZFC context.

\footnotetext{
${ }^{2}$ The main theorem of [Dav82], using Jensen coding, is that every transitive model of set theory has a class forcing extension in which $V=L(r)$ for a real $r$, such that $L_{\alpha}(r) \not \forall$ ZF for every ordinal $\alpha$. Since this situation is captured by a first-order theory which can hold in uncountable models, however, it cannot by itself imply pointwise definability; David apparently had in mind an appeal to further details of the proof. We note that in comparison to Jensen coding, our forcing is mild: it is progressively closed and can be made to preserve any of the usual large cardinals.
} 
Theorem 12 (Simpson). Let $\langle M, \in\rangle$ be a countable model of ZFC. Then, there is a class $U \subseteq M$ such that:

(i) $\langle M, \in, U\rangle$ satisfies ZFC in the language with a predicate for $U$.

(ii) Every element of $M$ is first-order definable in $\langle M, \in, U\rangle$.

Proof. Let us begin by enumerating the countable structure $M=$ $\left\{a_{n} \mid n<\omega\right\}$. Using the axiom of choice in $M$, it easy to see that every set $a_{n} \in M$ is coded in $M$ by a binary sequence, say $\bar{a}_{n} \in 2^{\alpha}$, for some ordinal $\alpha \in M$. Indeed, we can arrange things in such a way so that all of the relevant coding is done only on the even digits of $\bar{a}_{n}$, while all odd digits are 0 , except that the sequence $\bar{a}_{n}$ ends with two consecutive 1's immediately following a limit ordinal. These restrictions on codes will be useful when we concatenate many such sequences.

Consider the class forcing in $M$ to add a Cohen class of ordinals, that is, the partial order $\mathbb{Q}=2^{<\mathrm{ORD}}$ consisting of all ordinal length binary sequences, ordered by extension. Since $M$ is countable, we may enumerate the dense subclasses $\left\langle D_{n} \mid n<\omega\right\rangle$ of $\mathbb{Q}$ that are definable in $M$ from parameters. Let us suppose $D_{n}$ is defined by the formula $\varphi_{n}(x)$, using parameters from $M$, and by padding if necessary, we may assume that the parameters used in $\varphi_{n}$ are among $\left\{a_{j} \mid j<n\right\}$.

We now construct a certain $M$-generic filter for $\mathbb{Q}$ that will meet all the dense sets $D_{n}$ while simultaneously coding all the elements $a_{n}$ of $M$. Specifically, we recursively define a descending sequence of conditions $\left\langle p_{n} \mid n<\omega\right\rangle$ in $\mathbb{Q}$, beginning with $p_{0}=\emptyset$. At odd stages, let $p_{2 n+1}$ be a minimal-length extension of $p_{2 n}$ such that $p_{2 n+1} \in D_{n}$. At even stages, let $p_{2 n+2}=p_{2 n+1} \bar{a}_{n}$, the sequence obtained by concatenating the code $\bar{a}_{n}$ to the end of $p_{2 n+1}$. Finally, let $U=\bigcup_{n \in \omega} p_{n}$. This is the union of the filter consisting of the initial segments of $U$, which by construction is $M$-generic for $\mathbb{Q}$, since we met each dense class $D_{n}$. The forcing $\mathbb{Q}$ is $\kappa$-closed for every $\kappa$ in $M$, and so it follows by standard class-forcing arguments that we retain ZFC in the language with $U$ in the structure $\langle M, \in, U\rangle$.

A straightforward inductive argument now shows that every $a_{n}$ and $p_{n}$ is definable in this structure $\langle M, \in, U\rangle$. To see this, suppose that $p_{2 n}$ and $a_{j}$ for $j<n$ have already been defined. Then, $p_{2 n+1}$ is simply defined as the least initial segment $q$ of $U$ extending $p_{2 n}$ such that $M \models \varphi_{n}(q)$. Notice that $\varphi_{n}$ uses only parameters from $a_{j}$ for $j<n$, which have already been defined, so this definition can be carried out without the use of any parameters. Now, we define $p_{2 n+2}$ as the least initial segment $q$ of $U$, extending $p_{2 n+1}$, that ends in two consecutive 1's following a limit ordinal. Given $p_{2 n+1}$ and $p_{2 n+2}$, it is a trivial matter 
to define $\bar{a}_{n}$, and $a_{n}$ is easily defined as the unique set coded by $\bar{a}_{n}$. In this fashion, every element of the universe $M$ can be given a first-order parameter-free definition in the structure $\langle M, \in, U\rangle$.

In the proof of theorem 12, we took care in the selection of $U$ that the augmented structure $\langle M, \in, U\rangle$ would be pointwise definable. Let us briefly argue that such extra care is indeed required in general, for not every generic filter $U \subseteq 2^{<\mathrm{ORD}}$ over a countable structure $M$ need give rise to a pointwise definable structure $\langle M, \in, U\rangle$. One easy way to see this is simply to let $\left\langle N, \in^{*}, U^{*}\right\rangle$ be an internal ultrapower of $\langle M, \in, U\rangle$ by a nonprincipal ultrafilter on $\omega^{M}$. Since the definable elements must be in the range of this map, it follows that $\left\langle N, \in^{*}, U^{*}\right\rangle$ is not pointwise definable. But by elementarity, $U^{*}$ is $N$-generic for the forcing $2^{<\text {ORD }}$ in $N$. Alternatively, one may construct transitive counterexamples by starting with an uncountable $\langle M, \in\rangle \models \mathrm{ZFC}$, whose generic expansions $\langle M, \in, U\rangle$, obtained by forcing over $V$, remain uncountable and therefore not pointwise definable, but further forcing over $V$ can make them countable, while preserving the $M$-genericity of $U$. So extra care is indeed required. In general, of course, if one starts with a pointwise definable structure $M$, then any expansion $\langle M, \in, U\rangle$ of it will remain pointwise definable, and indeed, for this one only needs that $M$ is a Paris model (where every ordinal is definable without parameters), since generically $U$ will list every set of ordinals of $M$ and we will be able to define the beginning and ending points in $U$ of a code for any desired set.

We now use Simpson's result to prove theorem 11, where the pointwise definable structure is not obtained in an expansion of the original model $M$, by adding extra predicates to the language, but rather in an extension of $M$, obtained by forcing. Thus, we enlarge $M$ to a structure $M[G]$ in which every object is definable without parameters in the pure language of set theory.

Proof of theorem 11. Let $\langle M, \in\rangle$ be a countable model of ZFC. By forcing over $M$ if necessary, we may assume without loss of generality that $\langle M, \in\rangle \models \mathrm{GCH}$. By theorem 12, there is an amenable class $U \subseteq M$ so that every element $a \in M$ is first-order definable without parameters in $\langle M, \in, U\rangle$, which satisfies $\mathrm{ZFC}$ in the language with $U$. We now rid ourselves of the extra class $U$ by forcing to code it into the structure of the universe. Specifically, consider in $M$ the definable sequence of uncountable regular cardinals $\delta_{\alpha}=\aleph_{\omega \cdot \alpha+1}$, conveniently separated by large gaps that will avoid interference, and let $\mathbb{P}=\Pi_{\alpha \in U} \operatorname{Add}\left(\delta_{\alpha}, \delta_{\alpha}^{++}\right)$ be Easton's forcing, which forces failures of the GCH precisely at the cardinals $\delta_{\alpha}$ for $\alpha \in U$. If $G \subseteq \mathbb{P}$ is $M$-generic, then it is well known 
that $M[G] \models$ ZFC and all cardinals and cofinalities are preserved. It follows that the map $\alpha \mapsto \delta_{\alpha}$ is definable in $M[G]$, and so $U$ is definable in $M[G]$ by the equivalence $\alpha \in U$ if and only if $2^{\delta_{\alpha}}>\delta_{\alpha}^{+}$.

We shall now perform further forcing to ensure $V=$ HOD in a further extension $M[G][H]$, by coding into the GCH pattern at the regular cardinals $\gamma_{\alpha}=\aleph_{\omega \cdot \alpha+5}$, which sit conveniently in the gaps of the $\delta_{\alpha}$ sequence, again avoiding interference. Specifically, let $\mathbb{R}$ be the Easton support class-length forcing iteration, which at stage $\alpha$ generically decides either to force with trivial forcing, which will preserve the $\mathrm{GCH}$ at $\gamma_{\alpha}$, or with $\operatorname{Add}\left(\gamma_{\alpha}, \gamma_{\alpha}^{++}\right)$, which will violate the GCH at $\gamma_{\alpha}$. That is, the stage $\alpha$ forcing is the lottery sum $\{\overline{\mathbb{1}}\} \oplus \operatorname{Add}\left(\gamma_{\alpha}, \gamma_{\alpha}^{++}\right)$, which is the disjoint union of these partial orders joined by a new common upper bound $\mathbb{1}$, so that any generic filter picks exactly one factor and forces with it. Since the forcing at stage $\alpha$ is $<\gamma_{\alpha}$-closed, we may nicely factor the iteration $\mathbb{R}$ at arbitrarily large cardinals as set forcing followed by highly closed tail forcing, and so in the terminology of [Rei06] it is progressively closed and therefore preserves ZFC. Note also that $\mathbb{R}$ preserves all cardinals and cofinalities over $M[G]$. To see that $\mathbb{R}$ forces $V=$ HOD, we observe simply that every set of ordinals will eventually be coded into the GCH pattern on the coding points $\gamma_{\alpha}$, because we can extend any condition to a stronger condition that opts for the trivial or nontrivial side of the forcing in the correct pattern so as to code that set on an interval. Another way to say it is that the necessary bookkeeping is performed generically by a density argument. Specifically, suppose that $p \in \mathbb{R}$ and $\tau$ is a $\mathbb{R}$-name such that $p \Vdash \tau \subseteq \check{\gamma}$ for some ordinal $\gamma$. Let $\beta$ be large enough so that it is beyond the support of $p$, beyond $\gamma$ and also large enough so that $\tau$ is a $\mathbb{R}_{\beta}$-name. Extend $p$ to a stronger condition $q$, which for $\xi<\gamma$ opts for trivial forcing at stage $\beta+\xi$ with the same Boolean value as $\llbracket \check{\xi} \in \tau \rrbracket$ and for nontrivial forcing at stage $\beta+\xi$ with the Boolean value of $\llbracket \check{\xi} \notin \tau \rrbracket$. That is, $q(\beta+\xi)$ is an $\mathbb{R}_{\beta+\xi}$-name for the condition that chooses one way or the other in the lottery sum at that stage, depending on whether $\xi \in \tau_{H_{\beta}}$, where $H_{\beta}$ is the generic filter up to stage $\beta$. The condition $q$ therefore forces that the set named by $\tau$, whatever it is, will be coded into the GCH pattern of the $\gamma_{\beta+\xi}$ for $\xi<\gamma$. Thus, if $H \subseteq \mathbb{R}$ is $M$-generic, every set of ordinals in $M[G][H]$ will be ordinal-definable, and since every set can be easily coded by a set of ordinals, it follows that $M[G][H] \models V=$ HOD.

Let us now complete the argument. Since cardinals were preserved, it follows that the map $\alpha \mapsto \delta_{\alpha}$ is definable in $M[G][H]$. Since the $\mathbb{R}$ forcing only affects the GCH pattern at the $\gamma_{\alpha}$, it does not upset the coding we did in $M[G]$ at the $\delta_{\alpha}$, and so the class $U$ remains definable in 
$M[G][H]$ as the class of $\alpha$ for which the GCH fails at $\delta_{\alpha}$. Note that the class $M$ is definable from $U$ in $M[G][H]$ as the class of sets coded by a subsequence of $U$. Since $\langle M, \in, U\rangle$ is pointwise definable, it now follows that every element of $M$ is definable in $M[G][H]$ without parameters. In particular, every ordinal is definable in $M[G][H]$ without parameters. Since $M[G][H]$ is a model of $V=$ HOD, this implies that every element of $M[G][H]$ is definable without parameters.

One can easily combine the two steps of forcing in the proof of theorem 11 by interleaving them into one forcing iteration, which codes $U$ into the continuum pattern at $\delta_{\alpha}$ and holds lotteries at $\gamma_{\alpha}$, leading in the same way to a forcing extension satisfying $V=$ HOD in which $U$ is definable. We chose for clarity to separate the coding into two steps.

The argument can be easily modified to use other coding methods. For example, by using the $\diamond^{*}$ coding method as in [BT09] one may also achieve $\mathrm{GCH}$ in the final pointwise definable model.

Note also that the forcing of theorem 11 is very mild from the large cardinal perspective, for we first add a Cohen generic class of ordinals, and then code it into the GCH pattern while also forcing $V=$ HOD. These iterations are extremely nice, progressively closed Easton support iterations and can be made to have increasingly large gaps where no forcing occurs. Thus, they can easily be made to preserve any of the usual large cardinal notions. One may therefore find pointwise definable forcing extensions while preserving one's favorite large cardinals. For example, if $M$ has a supercompact cardinal $\kappa$, one should first make $\kappa$ Laver-indestructible, and then do all the coding above $\kappa$, which will preserve the supercompactness of $\kappa$ since it is $<\kappa$-directed closed.

\section{Extending the Result From ZFC TO GBC}

For the main contribution of this article, we should like now to extend the result from ZFC to Gödel-Bernays GBC set theory, which is a natural context for pointwise definability since it allows for a precise formal treatment of classes in set theory and many of the pointwise definability arguments have involved augmenting a model of set theory with additional proper classes, such as those arising with class forcing. We refer the reader to [Jec03] or [Men97, p. 225-86] for an overview of Gödel-Bernays set theory. Here, we regard a GBC model of set theory as a triple $\mathcal{M}=\langle M, S, \in\rangle$, consisting of a model $\langle M, \in\rangle$ of $\mathrm{ZFC}$ augmented with a family $S \subseteq P(M)$, whose members are the classes of $\mathcal{M}$, satisfying the $\mathrm{GBC}$ axioms. The $\mathrm{GBC}$ axioms extend the $\mathrm{ZFC}$ axioms to this two-sorted second-order context by allowing formulas in the replacement and separation axioms to make use of finitely many 
class parameters (but not to quantify over classes, a strengthening that constitutes Kelly-Morse set theory). In particular, if $\vec{X}$ is any finite list of classes from $S$, then the expansion $\langle M, \in, \vec{X}\rangle$, obtained by interpreting the classes in $\vec{X}$ as predicates over $M$, satisfies $\operatorname{ZFC}(\vec{X})$, the version of ZFC in which the classes of $\vec{X}$ may appear as atomic predicates in the replacement and separation axioms schemes, and furthermore, any class definable in $\langle M, \in, \vec{X}\rangle$ must be in $S$. In addition, GBC includes a global version of the axiom of choice, meaning that there is a single class choice function $F \in S$ such that $F(x) \in x$ for every nonempty set $x$.

Although the GBC axioms admit an elegant and economical formulation purely in terms of classes, as every model of GBC is determined entirely by its collection of classes, we prefer the two-sorted set-class formulation here so as to emphasize the connection with models of ZFC. In particular, any model $\langle M, \in\rangle$ of ZFC can be extended to a model $\langle M, S, \in\rangle$ of $\mathrm{GBC}$ by first finding an $M$-generic filter $G \subseteq \mathbb{Q}$ for the class forcing $\mathbb{Q}=M^{<\mathrm{ORD}}$ to add a global well-ordering of the universe - a forcing notion that is $\kappa$-closed for every cardinal $\kappa$ and hence adds no sets - and then letting $S$ consist of the classes definable in $\langle M, \in, G\rangle$ from parameters; the forcing to add $G$ ensures global choice in $\langle M, S, \in\rangle$, and is unnecessary if $\langle M, \in\rangle$ already has a global choice class function. It follows that GBC is conservative over ZFC in the sense that any statement purely about sets that is provable in GBC is also provable in ZFC.

The method of forcing works over models of GBC just as it does over models of ZFC. In particular, if $\mathbb{P}$ is a partial order in a model $\mathcal{M}=\langle M, S, \in\rangle$ of $\mathrm{GBC}$ and $G \subseteq \mathbb{P}$ is $\mathcal{M}$-generic, then we may define $\mathcal{M}[G]=\langle M[G], S[G], \in\rangle$, where $M[G]$ and $S[G]$ consist of interpreting in the usual way via $G$ the sets and classes that are $\mathbb{P}$-names in $\mathcal{M}$. In the case of set forcing, where $\mathbb{P} \in M$, the analogues of the basic ZFC forcing lemmas hold also for GBC, and in this case the forcing extension $\mathcal{M}[G]$ continues to satisfy GBC. Class forcing, in contrast, can destroy GBC just as it can destroy ZFC, such as by making every set countable, to give one badly behaved example. Nevertheless, there are large families of nicely behaved class-forcing notions that necessarily preserve GBC. This includes progressively closed forcing in the sense of [Rei06], that is, forcing notions that factor for arbitrarily large cardinals $\kappa$ as $\mathbb{P}_{0} * \mathbb{P}_{\text {tail }}$, where $\mathbb{P}_{0}$ is a set and $\mathbb{P}_{\text {tail }}$ is $<\kappa$-closed. As it happens, all of the forcing notions necessary to carry out the constructions in this article are progressively closed, and so the corresponding forcing extensions will all satisfy GBC. 
The main theorem of this section is that every countable model of Gödel-Bernays set theory can be extended to a pointwise definable model.

Theorem 13. Every countable model of Gödel-Bernays set theory has a pointwise definable extension, where every set and class is first-order definable without parameters.

Thus, the original countable GBC model $\langle M, S, \in\rangle$ is extended to a pointwise-definable ZFC model $\langle M[G], \in\rangle$, such that every set in $M[G]$ and every class in $S$ is definable in $\langle M[G], \in\rangle$ without parameters.

In order to prove this theorem, we shall first prove a special case of it, the case where the original model $\langle M, S, \in\rangle$ is principal, meaning that there is a class $X \in S$ such that every class in $S$ is firstorder definable in $\langle M, \in, X\rangle$ with set parameters. The principal models of GBC include many natural instances. For example, earlier we explained that any model of ZFC can be transformed into a GBC model with the same sets, by first forcing global choice and then augmenting with the classes definable from that generic well-ordering; the resulting GBC model is principal by construction. Furthermore, the collection of principal models of GBC is closed under forcing, even proper class forcing (provided GBC itself is preserved), since one can amalgamate the original generating class with the newly generic class to form a new generating class. So we have plenty of principal models of GBC. Nevertheless, it is also easy to construct non-principal models, such as the weak limit of a suitable iteration of $\omega$ many extensions $M \subseteq M\left[G_{0}\right] \subseteq M\left[G_{0}\right]\left[G_{1}\right] \subseteq \cdots$, whose union satisfies GBC but is nonprincipal. Additional non-principal models are provided by the fact that no model $\langle M, S, \in\rangle$ of Kelly-Morse set theory can be principal as a GBC model, since KM proves that every class $X$ admits a first-order truth class for $\langle M, \in, X\rangle$, since for each $n$ the $\sum_{\sim}(X)$ truth class is unique satisfying the recursive definition of truth and these classes can be unified into one class in KM, but no such truth class can be first-order definable from $X$ over $M$. An instance of this arises from an inaccessible cardinal $\kappa$ with the structure $\left\langle V_{\kappa}, V_{\kappa+1}, \in\right\rangle$, which is a model of GBC and in fact KM, but is easily seen not to be principal as a GBC model on cardinality grounds, since it has $2^{\kappa}$ many classes but only $\kappa$ many sets and hence only $\kappa$ many classes definable from any fixed class. Nevertheless, this model can be made principal by the forcing to collapse $2^{\kappa}$ to $\kappa$, which adds no sets to $V_{\kappa}$, but which adds a generic $\kappa$-enumeration of the ground model subsets of $V_{\kappa}$. 
Theorem 14. Every countable principal GBC model has a class forcing extension that is pointwise definable, in which every set and class is first-order definable without parameters.

Proof. Suppose that $\mathcal{M}=\langle M, S, \in\rangle$ is a principal countable model of GBC. To prove the theorem we must show there is a class forcing extension $\langle M[G], \in\rangle$, satisfying ZFC such that every set in $M[G]$ and every class in $S$ is definable in $\langle M[G], \in\rangle$ without parameters. The proof will follow the proof of theorem 11, extending the argument to handle the issues arising on account of the second-order part of the models. Since $\mathcal{M}$ is principal, there is a class $X \in S$ such that every class in $S$ is first-order definable in the structure $\langle M, \in, X\rangle$. By forcing if necessary, we may assume $\mathcal{M} \models \mathrm{GCH}$.

The first step is to prove the analogue of Simpson's theorem for this case. Specifically, we claim that there is an amenable class $U$ such that $\langle M, \in, U\rangle$ satisfies $\operatorname{ZFC}(U)$ and every set and class in $\langle M, S, \in\rangle$ is definable in $\langle M, \in, U\rangle$ without parameters. The proof is just as in theorem 12, with an extra step. Enumerate $M=\left\langle a_{n} \mid n<\omega\right\rangle$ and choose codes $\tilde{a}_{n} \in 2^{<\mathrm{ORD}}$ for each $a_{n}$. Let $\mathbb{Q}=2^{<\mathrm{ORD}}$ and construct an $\mathcal{M}$-generic class $U_{0} \subset \mathbb{Q}$ as in the proof of theorem 12 so that every set in $M$ is definable without parameters in $\left\langle M, \in, U_{0}\right\rangle$. Since the forcing is $\kappa$-closed for every cardinal $\kappa$, it adds no sets and $\mathcal{M}\left[U_{0}\right]=\left\langle M, S\left[U_{0}\right], \in\right\rangle \models$ GBC, where $S\left[U_{0}\right]$ is the collection of classes obtained by interpreting via $U_{0}$ the $\mathbb{Q}$-names in $\mathcal{M}$. Let $U$ be the class $X \oplus U_{0}$, combining the two classes in some canonical manner as a class of ordinals, and observe that $\langle M, \in, U\rangle$ satisfies $\operatorname{ZFC}(U)$, because the forcing $\mathbb{Q}$ is progressively closed - indeed, it is $\kappa$-closed for every cardinal $\kappa$. Since $U_{0}$ is definable from $U$ in $\langle M, \in, U\rangle$, it follows that every element of $M$ is definable in $\langle M, \in, U\rangle$ without parameters. And since $X$ is also definable from $U$ there, and every class in $S$ is definable from $X$ with set parameters, it follows that every class in $S$ is also definable in $\langle M, \in, U\rangle$ without parameters, as desired.

As in theorem 11, we may now force to code the digits of $U$ into the GCH pattern at the cardinals $\delta_{\alpha}=\aleph_{\omega \cdot \alpha+1}$, producing a forcing extension $M[G]$ in which $U$ is definable without parameters. It follows that $M$ is also definable there, since the sets in $M$ are exactly the sets that are coded into a block of $U_{0}$. Next, a further forcing extension $M[G][H]$ satisfies $V=$ HOD by generically coding the GCH pattern at the cardinals $\gamma_{\alpha}=\aleph_{\omega \cdot \alpha+5}$ as before. Thus, every element of $M[G][H]$ is definable from ordinal parameters, but these are definable without parameters since every element of $M$, including every ordinal, is definable from $M$ and $U_{0}$, which are definable from $U$, which is definable 
without parameters in $\langle M[G][H], \in\rangle$. It follows that every class in $S$ is definable without parameters in $\langle M[G][H], \in\rangle$, since $X$ is definable from $U$ there, and $U$ is definable without parameters. So every set and class in $\langle M[G][H], \in\rangle$ is definable without parameters, as desired.

To complete the proof of theorem 13, it suffices to show that every countable GBC model $\mathcal{M}$ can be extended to a principal GBC model. Our initial attempts to prove this involved meta-class forcing. We wanted to replicate the situation of the model $\left\langle V_{\kappa}, V_{\kappa+1}, \in\right\rangle$ in the case $\kappa$ is an inaccessible cardinal, since this structure is non-principal, but is made principle in the forcing extension $V[G]$, where $G \subseteq \operatorname{Coll}\left(\kappa, V_{\kappa+1}\right)$, which collapses $2^{\kappa}$ to $\kappa$. The generic filter $G$ can be viewed as a subclass of $\kappa \times V_{\kappa}$, whose columns are exactly the ground model subsets of $V_{\kappa}$, which are exactly the classes of the original model. In this way, the old classes are unified into one new generic class. The forcing in this argument is meta-class forcing, as opposed to merely class forcing, since the individual conditions are classes in $\left\langle V_{\kappa}, V_{\kappa+1}, \in\right\rangle$.

A similar idea appears to work over any model of Kelly-Morse set theory, although the details of verifying the meta-class forcing technology are abundant. The idea is that given a model $\langle M, S, \in\rangle \models \mathrm{KM}$, one considers the meta-class of conditions consisting of an ordinal $\alpha$ and a subclass $A \subseteq \alpha \times M$, with $A \in S$. Stronger conditions enlarge $\alpha$ and specify additional columns, and so the forcing is exactly analogous to Coll(ORD $\left.{ }^{M}, S\right)$ as above. In this way, it is dense to add any class of $S$ onto a vertical slice, and the generic class $G \subseteq \mathrm{ORD} \times M$ will unify all the classes $X \in S$ into one class, making a principal model.

Such a meta-class forcing method, however, does not seem to work easily over GBC models, and one should not expect to add a single generic class from which all the classes of $S$ are uniformly definable, by varying only set parameters.

Nevertheless, we avoid the difficulties of these higher-order forcing arguments and complete the proof of theorem 13 by appealing to the following remarkable observation of Sy Friedman (sketched in an email correspondence with the first author), which shows that every countable GBC model does indeed have a principal extension with the same sets. Kossak and Schmerl prove a similar fact about models of PA in [KS06, Theorem 6.5.6], showing that for any model $\left\langle M, R_{0}, R_{1}, \ldots\right\rangle \models$ PA* there is a $G \subseteq M$ such that $\langle M, G\rangle \models \mathrm{PA}^{*}$ and each $R_{i}$ is definable in $\langle M, G\rangle$. It is the Kossak/Schmerl proof method that we shall adapt to GBC models here. Viewing the original Kossak/Schmerl theorem as one about models of $\mathrm{GBC} \neg \infty$, the theory $\mathrm{GBC}$ with the infinity axiom replaced by its negation (and augmented with the assertion that every 
set has a transitive closure), Ali Enayat has described theorem 15 here as one of the positive instances where a model-theoretic theorem about $\mathrm{GBC}^{\urcorner \infty}$ extends to GBC; not all of them do.

Theorem 15 (Friedman/Kossak/Schmerl). Every countable model $\mathcal{M}$ of $\mathrm{GBC}$ can be extended to a principal model $\mathcal{M}[Y] \models \mathrm{GBC}$ while adding no sets, only classes. Indeed, in the extension, every set and class in $\mathcal{M}$ is definable in the structure $\langle M, \in, Y\rangle$.

Proof. For clarity, let us mention up-front that the extension $\mathcal{M}[Y]$ we produce will not necessarily be a class forcing extension of $\mathcal{M}$, although it will satisfy GBC because of the close connections it has with a sequence of increasingly partially generic extensions. Specifically, for each $n$ we will be able to realize $\mathcal{M}[Y]$ as a $\Sigma_{n}$-generic extension of $\mathcal{M}$ by a partial order $\mathbb{Q}_{n}$, meaning that all $\sum_{\sim}$ definable dense subclasses of $\mathbb{Q}_{n}$ are met by the filter, and by increasing $n$ this suffices to capture the whole of GBC.

Suppose that $\mathcal{M}=\langle M, S, \in\rangle$ is a countable model of GBC, meaning that it has countably many sets and classes. Enumerate the classes of ordinals in $S$ as $\left\langle A_{n} \mid n<\omega\right\rangle$, so that $A_{n} \subseteq \mathrm{ORD}^{M}$ and every class in $S$ is first-order definable from some $A_{n}$. We shall add a class $Y$ of ordinals in such a way that $\langle M, \in, Y\rangle$ satisfies $\mathrm{ZFC}(Y)$ and every $A_{n}$ is coded into $Y$, hidden away by coding on increasingly difficult-to-define subclasses of ordinals. Specifically, we will construct a sequence of trees $\mathbb{Q}_{0} \supset \mathbb{Q}_{1} \supset \mathbb{Q}_{2} \supset \cdots$, each definable but with increasingly complex definitions. The class $Y$ will give a branch through $\bigcap \mathbb{Q}_{n}$, and $A_{n}$ will be determined by the choices that $Y$ makes within $\mathbb{Q}_{n}$. Thus $A_{n}$ will be recoverable from $Y$ and $\mathbb{Q}_{n}$, but the increasing complexity of $\mathbb{Q}_{n}$ suggests that we should not expect a uniform definition.

If we succeed, then it follows by induction that every $A_{n}$ and hence every class in $S$, is definable in $\langle M, \in, Y\rangle$, whose definable classes form a principal GBC model, and so we will have proved the theorem.

The argument will rely on some refinements of the customary general facts about class forcing, which we shall summarize here but not prove. First, for any sufficiently nice ${ }^{3}$ class forcing notion $\mathbb{P}$, the forcing relation $p \Vdash_{\mathbb{P}} \varphi$ is definable in $M$ from $\mathbb{P}$; the refinement we need is that for $\varphi$ of bounded complexity $\Sigma_{n}$, the forcing relation has some bounded complexity $\Sigma_{k}(\mathbb{P})$ in $M$. Second, whenever $p \Vdash_{\mathbb{P}} \varphi$, then $M[G] \models \varphi$ for any $M$-generic filter $G \subseteq \mathbb{P}$ containing $p$; the refinement we need

\footnotetext{
${ }^{3}$ The question of whether every class forcing notion has a definable forcing relation (i.e. 'is sufficiently nice') is open. However, a sufficient condition which holds of a great many class forcings, including all those appearing in this paper, is that every subset $A \subset \mathbb{P}$ is contained in a complete subposet $Q \subset_{C} \mathbb{P}$
} 
is that for $\varphi$ of bounded complexity $\Sigma_{n}$, there is some $k$ such that $M[G] \models \varphi$ for any $\Sigma_{k}(\mathbb{P})$-generic filter $G \subseteq \mathbb{P}$ containing $p$. Third, whenever $M[G] \models \varphi$ for some $M$-generic filter $G \subseteq \mathbb{P}$, then there is a condition $p \in G$ such that $p \Vdash_{\mathbb{P}} \varphi$; the refinement we need is that for $\varphi$ of bounded complexity $\Sigma_{n}$, there is some $k$ such that whenever $M[G] \models \varphi$ for some $\Sigma_{k}(\mathbb{P})$-generic filter $G \subseteq \mathbb{P}$, then $p \Vdash_{\mathbb{P}} \varphi$ for some $p \in G$. These refinements simply assert that the forcing mechanics are sound with respect to partially but sufficiently generic filters, and can be proved by induction by following the usual proofs of the forcing lemmas and paying attention to the complexity of the dense sets that arise in the arguments. It is not relevant for our application to find the strictly optimal relations between $n$ and $k$, although one could do this.

Now, let us return to the argument and create the generic coding class $Y$. Let $\mathbb{Q}_{0}=\operatorname{Add}(\mathrm{ORD}, 1)=2^{<\mathrm{ORD}}$ be the class of all binary ordinal length sequences, ordered by extension. We will construct a descending sequence of class forcing notions

$$
\mathbb{Q}_{0} \supset \mathbb{Q}_{1} \supset \mathbb{Q}_{2} \supset \cdots
$$

Each $\mathbb{Q}_{n}$ will be a perfect tree, that is, a subclass of $2^{<\mathrm{ORD}}$ that is splitting (any node can be extended to two incompatible nodes, though not necessarily at the next level) and contains full limits. There is a canonical embedding of $2^{<\mathrm{ORD}}$ into any perfect tree, whose image is exactly the splitting nodes (nodes with two immediate successors) of the tree. This embedding is dense, and therefore witnesses the forcing equivalence of $\operatorname{Add}(\mathrm{ORD}, 1)$ and any perfect tree. Thus, all the $\mathbb{Q}_{n}$ are individually forcing equivalent to $\operatorname{Add}(\mathrm{ORD}, 1)$, which is $\kappa$-closed for every cardinal $\kappa$ and therefore adds no new sets, while forcing ZFC relative to the generic class.

Given $\mathbb{Q}_{n}$, we construct $\mathbb{Q}_{n+1}$ in two stages. First, we will refine $\mathbb{Q}_{n}$ to a subtree with the property that any branch will meet all $\sum_{n}$ definable dense subclasses of $\mathbb{Q}_{n}$. By increasing the length of the stem, or portion of the tree below the first branch point, in this stage we will also guarantee a branch through the intersection. We then further refine this subtree to obtain $\mathbb{Q}_{n+1}$, ensuring that any branch through $\mathbb{Q}_{n+1}$ codes $A_{n}$.

The construction proceeds as follows. We fix a sequence of ordinals $\left\langle a_{n} \mid n \in \omega\right\rangle$ cofinal in $\mathcal{M}$ (these will govern the growth of the stems of the $\left.\mathbb{Q}_{n}\right)$. Given $\mathbb{Q}_{n}$, we fix an enumeration $\left\langle D_{\alpha} \mid \alpha \in \mathrm{ORD}\right\rangle$ in $\mathcal{M}$ of all $\sum_{\sim}\left(\mathbb{Q}_{n}\right)$ definable dense subclasses of $\mathbb{Q}_{n}$. We now construct an embedding $f: 2^{<\text {ORD }} \rightarrow \mathbb{Q}_{n}$. Let $f(\emptyset)$ be the least splitting node of $\mathbb{Q}_{n}$ which has length exceeding $a_{n}$ (by 'least' we mean the node of shortest length, and if more than one such node exists we take the least 
according to some fixed class well-ordering of $\mathcal{M}$ from $S)$. Given $f(p)$ where $p$ has length $\alpha$, and fixing $e \in\{0,1\}$, we let $f\left(p^{\curvearrowleft} e\right)$ be the least splitting node $q$ of $\mathbb{Q}_{n}$ extending $f(p)^{\wedge} e$ such that $q \in D_{\alpha}$. For $p$ of limit length, we take $f(p)$ to be the least splitting node extending $\bigcup_{p^{\prime} \subset p} f\left(p^{\prime}\right)$ and lying in $D_{\alpha}$. Let $\mathbb{Q}_{n}^{*}$ denote the subtree of $\mathbb{Q}_{n}$ determined by $f$, that is, the tree consisting of all predecessors of members of $\operatorname{ran}(f)$. For each $\alpha$, any branch through $\mathbb{Q}_{n}^{*}$ will necessarily meet $\{f(p) \mid p$ has length $\alpha\} \subset D_{\alpha}$, and so any branch will meet all $\sum_{\sim}$ definable dense subclasses of $\mathbb{Q}_{n}$.

We now refine $\mathbb{Q}_{n}^{*}$ to encode $A_{n}$ by selecting only those $q \in \mathbb{Q}_{n}^{*}$ which "choose according to $A_{n}$ on the even splitting nodes." That is, we select those $q$ such that, for any predecessor $q^{\prime}$ of $q$, if $q^{\prime}$ is a splitting node of $\mathbb{Q}_{n}^{*}$ with exactly $2 \cdot \alpha$ many splitting nodes preceding it, then $q^{\prime} \frown 1 \in q$ if and only if $\alpha \in A_{n}$. Let $\mathbb{Q}_{n+1}$ be the subtree of $\mathbb{Q}_{n}^{*}$ determined by such $q$, that is, the tree consisting of all predecessors of such $q$. Given $\mathbb{Q}_{n}$ together with any branch of $\mathbb{Q}_{n+1}$ we can easily recover $A_{n}$ by comparing values of the branch corresponding to the even splitting nodes of $\mathbb{Q}_{n}$. Conversely, note that $\mathbb{Q}_{n+1}$ was defined from parameters $\mathbb{Q}_{n}$ and $A_{n}$.

This completes the construction. We now take $Y=\bigcap \mathbb{Q}_{n}$. That $Y$ is nonempty and contains a single branch follows from the fact the $\mathbb{Q}_{n}$ are nested and have stems of increasing length unbounded in $\mathrm{ORD}^{\mathcal{M}}$. The key observation remaining is that the construction ensures that $\langle M, \in, Y\rangle$ satisfies $\mathrm{ZFC}(Y)$. Any axiom of $\mathrm{ZFC}(Y)$ has some logical complexity $\Sigma_{n}$ in the forcing language, and by the refined forcing facts mentioned above there is some $k_{n}$ sufficiently large so that the forcing lemmas concerning $\Sigma_{n}$ work as expected for all $\Sigma_{k_{n}}\left(\mathbb{Q}_{k_{n}}\right)$ generic filters of $\mathbb{Q}_{k_{n}}$. By construction $Y$ has the required level of genericity for $\mathbb{Q}_{k_{n}}$, and so $\langle M, \in, Y\rangle$ satisfies every axiom of $\mathrm{ZFC}(Y)$, as desired. As we have observed, each $A_{n}$ is definable from $Y$ and $\mathbb{Q}_{n}$. The partial order $\mathbb{Q}_{n+1}$ is definable from $\mathbb{Q}_{n}$ and $A_{n}$, and it follows inductively that each $A_{n}$ and each $\mathbb{Q}_{n}$ are definable in $\langle M, \in, Y\rangle$. Thus every class in $S$ is definable from $Y$, and so we have produced the desired principal model $\langle M, \in, Y\rangle$ extending $\mathcal{M}$. In particular, every set and class of this model is definable using only the parameter $Y$ over $M$.

Theorem 13 now follows from theorems 14 and 15, since any countable model of GBC can first be made principal by theorem 15 and then pointwise definable by theorem 14. In fact, the Friedman/Kossak/Schmerl theorem (theorem 15) can also serve as a replacement for Simpson's theorem 12 in the proof of theorem 11, since as we mentioned every set 
and class of $\mathcal{M}$ is definable in the structure $\langle M, \in, Y\rangle$, which was the whole point of theorem 12 .

Finally, we remark that the proof we have just given of theorem 13 allows us to preserve any of the usual large cardinals from the ground model to the pointwise definable extension. This is because the application of theorem 15 does not add sets, and so preserves all large cardinal properties, and the remaining forcing of theorem 14 is the very nice, progressively closed Easton-support forcing iteration to code the class $Y$ and then force $V=$ HOD. As we mentioned after theorem 11 , this can be easily arranged to accommodate any of the usual large cardinal notions. Thus, any countable model of GBC with any of the usual large cardinals has an extension to a pointwise-definable model of ZFC, in which every set and class is definable without parameters, and in which those large cardinals are preserved.

\section{REFERENCES}

[Ani] Username Anixx. Is analysis as taught in universities in fact the analysis of definable numbers? MathOverflow. http://mathoverflow. net/questions/44102 (2010-10-29).

[BT09] Andrew Brooke-Taylor. Large cardinals and definable well-orders on the universe. Journal of Symbolic Logic, 74(2):641-654, 2009.

[Dav82] R. David. Some applications of Jensen's coding theorem. Ann. Math. Logic, 22(2):177-196, 1982.

[Ena02] Ali Enayat. Counting models of set theory. Fund. Math., 174(1):23-47, 2002.

[Ena05] Ali Enayat. Models of set theory with definable ordinals. Archive of Mathematical Logic, 44:363-385, 2005.

[FHR] Gunter Fuchs, Joel David Hamkins, and Jonas Reitz. Set-theoretic geology. in preparation.

[HJ] Joel David Hamkins and Thomas Johnstone. The Resurrection Axioms. in preparation.

[Jec03] Thomas Jech. Set Theory. Springer Monographs in Mathematics, 3rd edition, 2003.

[KS06] Roman Kossak and James Schmerl. The Structure of Models of Peano Arithmetic, volume 50 of Oxford Logic Guides. Oxford University Press, Oxford, 2006.

[Men97] Elliott Mendelson. An Introduction to Mathematical Logic. Chapman \& Hall, London, 4th edition, 1997.

[Myh52] John Myhill. The hypothesis that all classes are nameable. Proc. Nat. Acad. Sci. U. S. A., 38:979-981, 1952.

[Rei06] Jonas Reitz. The Ground Axiom. PhD thesis, The Graduate Center of the City University of New York, September 2006.

[Sim74] S. Simpson. Forcing and models of arithmetic. Proceeding of the American Mathematical Society, 43:93-194, 1974. 
J. D. Hamkins, Mathematics, The Graduate Center of The City University of New York, 365 Fifth Avenue, New York, NY 10016 \& Mathematics, The College of Staten Island of CUNY, Staten Island, NY 10314

E-mail address: jhamkins@gc.cuny.edu, http://jdh.hamkins.org

D. Linetsky, Mathematics, The Graduate Center of The City University of New York, 365 Fifth Avenue, New York, NY 10016

E-mail address: dlinetsky@gmail.com

J. Reitz, New York City College of Technology of The City University of New York, Mathematics, 300 Jay Street, Brooklyn, NY 11201

E-mail address: jonasreitz@gmail.com 